Sportwiss 2013 $\cdot 43: 85-89$

DOI 10.1007/s12662-013-0281-0

Online publiziert: 7. März 2013

๑) Springer-Verlag Berlin Heidelberg 2013

Martin Lames ${ }^{1}$. Mark Pfeiffer ${ }^{2}$. Andreas Hohmann ${ }^{3}$. Andrea Horn ${ }^{4}$

${ }^{1}$ Lehrstuhl für Trainingswissenschaft und Sportinformatik, München, Deutschland

${ }^{2}$ Abteilung Theorie und Praxis der Sportarten, FB 02, Institut für Sportwissenschaft,

Johannes Gutenberg-Universität Mainz, Mainz, Deutschland

${ }^{3}$ Institut für Sportwissenschaft, Arbeitsbereich I Trainings- und Bewegungswissenschaft, Universität Bayreuth, Bayreuth, Deutschland

${ }^{4}$ Fachbereich Forschung und Entwicklung, Fachgebiet Trainingswissenschaft,

Bundesinstitut für Sportwissenschaft, Bonn, Deutschland

\title{
Erklärung zur Lage der universitären
} Trainingswissenschaft

\section{Situation der Trainingswissenschaft}

Nachgang zweier Veranstaltungen der dvs-Sektion Trainingswissenschaft, dem dvs-Workshop „Kernspin und Topspin - Das Spektrum der universitären Trainingswissenschaft" 2011 in München und der Sektionstagung 2012 in Halle. Mit ihr werden die zentralen Inhalte und Ergebnisse der bisherigen Gespräche einem größeren Interessentenkreis zugänglich gemacht. Gleichzeitig soll die Erklärung eine vertiefende Diskussion über das Selbstverständnis der Trainingswissenschaft anregen. Dabei ist den Autoren bewusst, dass an der einen oder anderen Stelle die Komplexität des Sachverhalts eine inhaltlich tiefergehende Ausdifferenzierung und weiterführende Diskussion verlangt hätte. Im Hinblick auf die genannte Zielstellung wurde hierauf zugunsten eines vertretbaren Umfangs verzichtet. Ferner ist darauf hinzuweisen, dass eine Situationsbeschreibung in Form einer Erklärung nicht frei von den persönlichen Erfahrungen der „sich Erklärenden“ sein kann. Die nachfolgenden Ausführungen beanspruchen somit weder einen Anspruch auf Allgemeingültigkeit noch auf Vollständigkeit. Es wird daher grundsätzlich begrüßt, wenn von verschiedener Stelle weitere Positionen, Standpunkte oder Erfahrungen in die weiteren Diskussionen eingebracht werden.
Die Trainingswissenschaft ist eine der jüngeren Disziplinen in der Deutschen Vereinigung für Sportwissenschaft (dvs). Erst 1992 wurde eine eigenständige Sektion "Trainingswissenschaft" eingerichtet, die sich aus der Gründungs-Sektion „Bewegung und Training" ausdifferenziert hat. Von der erfolgreichen Sektionsarbeit und einem grundlegenden Interesse an trainingswissenschaftlichen Themen zeugen die seither durchgeführten 10 Symposien und 7 Workshops der dvs-Sektion Trainingswissenschaft. Dazu kommen 8 gemeinsam mit den dvs-Sektionen Biomechanik und Sportmotorik ausgerichtete Symposien.

Auch die Institutionalisierung der Trainingswissenschaft an den sportwissenschaftlichen Einrichtungen der Universitäten kann als zufriedenstellend bezeichnet werden. An über 30 der 64 sportwissenschaftlichen Hochschuleinrichtungen (Universitäten und Fachhochschulen bzw. Pädagogischen Hochschulen) in Deutschland führen Lehrstühle und Professuren einen der Begriffe „Trainingswissenschaft", „Training“ oder „Trainingslehre" in ihrer Denomination. Diesen Grad an institutioneller Verankerung in den Lehrkörpern kann neben der Sportpädagogik kaum eine andere Disziplin für sich reklamieren.
An den sportwissenschaftlichen Hochschuleinrichtungen in Deutschland hat sich die Trainingswissenschaft als eigenständiges Lehrgebiet fest etabliert. In den Curricula sowohl der Lehramtsstudiengänge als auch der vielfältigen Bachelorund Masterstudiengänge gehören trainingswissenschaftliche Themen und Inhalte zum festen Bestandteil. Darüber hinaus wird trainingswissenschaftliches Wissen, vordergründig zu den Gegenstandsbereichen sportliches Training und sportliche Leistungsfähigkeit, im Rahmen disziplinübergreifender Veranstaltungen, wie z. B. zu verschiedenen Handlungsfeldern des Sports (Schulsport, Rehabilitationssport, Fitness- und Breitensport) vermittelt. Im Bereich der sportpraktischen Ausbildung nehmen trainingswissenschaftliche Inhalte traditionell einen groBen Stellenwert bei der Vermittlung theoretischer und methodischer Grundlagen ein, wobei die Übertragung wissenschaftlich fundierter Erkenntnisse auf die sportartspezifischen Besonderheiten im Mittelpunkt steht.

Man kann also bereits an dieser Stelle festhalten, dass es eine etablierte sportwissenschaftliche Disziplin Trainingswissenschaft gibt, deren Markenkern kurz wie folgt umschrieben werden kann:

- Ihr Selbstverständnis besteht in der wissenschaftlichen Fundierung von praktischem Handeln im Sport, und zwar aus einer ganzheitlichen und angewandten Perspektive. 
- Sie integriert dazu Beiträge verschiedener Wissenschaftsdisziplinen, steuert eigenes Grundlagen- und Anwendungswissen zur Domain „Training" bei, sucht experimentell nach optimalen Trainingsmethoden und begleitet wissenschaftlich das praktische Handeln im Sport.

- Ihr klassisches Anwendungsfeld ist der Leistungssport, auf der Basis des offenen Trainingsbegriffs leistet sie aber auch Beiträge in allen weiteren Anwendungsgebieten des Sports.

Mit diesem Programm hat sich die Trainingswissenschaft neben der Sportpädagogik/Sportdidaktik und der Sportmedizin als ein Kernstück der Sportwissenschaft und somit als Eckpfeiler der Grundausstattung eines universitären Sportinstituts etabliert. Diese Eckposition hat sich insbesondere aus dem Alleinstellungsmerkmal abgeleitet, dass die Trainingswissenschaft als einzige sportwissenschaftliche Disziplin eine dominante Anwendungsorientierung reklamiert und als „Wissenschaft der sportlichen Interventionen" bezeichnet werden kann. Den wichtigsten Ausgangspunkt trainingswissenschaftlicher Fragestellungen stellt der Handlungsprozess des sportlichen Trainings dar. Dieser soll im Sinne der Herrmann'schen Domain-Forschung wissenschaftlich bearbeitet werden. Auch in der Zielperspektive trainingswissenschaftlicher Forschung nimmt die Umsetzbarkeit in der Trainingspraxis eine führende Stellung ein.

Auch wenn es hier primär um die Lage der Trainingswissenschaft an den Universitäten gehen soll, so darf im Zusammenhang dieser Bestandsaufnahme dennoch auch aus organisationssoziologischer Sicht mit Genugtuung darauf hingewiesen werden, dass es die Trainingswissenschaft nach der Sportmedizin wohl am ehesten erreicht hat, sich mit akademisch geprägten Einrichtungen auch außerhalb der Universitäten zu etablieren. Neben den 22 Olympiastützpunkten zeigt sich dies am deutlichsten am Leipziger Institut für Angewandte Trainingswissenschaft (IAT), das seine trainingswissenschaftlich eingefärbte Corporate Identity bereits durch die prägnante Namensgebung dokumentiert.
Ungeachtet dieser nach wie vor marktfähigen Positionierung geben die in den vergangenen rund 20 Jahren vor sich gegangenen gravierenden Veränderungen im Bereich des Sports und auch der Universitäten und der Wissenschaft Anlass zu Reflektionen über die Zukunftsfähigkeit der Disziplin, die in dieser Erklärung festgehalten werden sollen. Insbesondere im Zuge des „Bologna-Prozesses“ wurde eine umfassende Umstrukturierung von Studiengängen angestoßen. Die dabei etablierten Studiengangsprofilierungen, wie z. B. in Richtung der Gesundheitsförderung und Rehabilitation oder insbesondere des Leistungs- und Spitzensports, bringen für die universitäre Trainingswissenschaft nicht nur inhaltliche Chancen, sondern u. U. auch Risiken oder schwer einlösbare akademische Anforderungen mit sich.

Ganz allgemein betrachtet, hat die flächendeckende Neukonzeption von Bachelor- und Masterstudiengängen eine inhaltliche Profilierung nicht nur der Studienangebote, sondern auch der Arbeitsund Forschungsschwerpunkte an den Sportinstituten selbst mit sich gebracht, die natürlich auch mit Strukturentscheidungen einhergingen. Es ist nachvollziehbar, dass diese Prozesse häufig zu einer Abkehr von der Grundstruktur der Sportinstitute geführt haben und führen werden, von der die sog. Eckprofessuren der empfohlenen Mindestausstattung - darunter auch die für die Trainings- (und ggf. Bewegungs-)Wissenschaft - betroffen sind (vgl. „Memorandum zur Entwicklung der Sportwissenschaft"). Die Trainingswissenschaft ist daher dringend angehalten, ihre Beitragsfähigkeit auch unter dem Dach von solchen Institutionen nachzuweisen, die sich weniger einer Sport- als vielmehr einer Bewegungs- oder Gesundheitsorientierung verschrieben haben. Auch in diesem Kontext dürfte die Trainingswissenschaft aufgrund ihrer Fokussierung auf die Nachhaltigkeit von Ansteuerungsmaßnahmen jedweder Art über ausgezeichnete Argumente und Instrumente verfügen.

Über die inhaltliche Profilierung hinaus betreffen die akademischen Herausforderungen vor allem die leistungsbasierten Steuerungsinstrumente, die seit Ende der 1990er Jahre im Bereich der
Hochschulverwaltung eingeführt wurden. Neben der schon bisher überwiegend praktizierten Erfassung der Lehrleistungen werden hierfür eine Reihe formal objektivierbarer Indikatoren der Forschungsleistung einer Quantifizierung unterzogen, die sich auf Kriterien wie die Höhe der Drittmitteleinwerbungen, die Publikation der Forschungsergebnisse und die Qualifizierung von wissenschaftlichem Nachwuchs beruft. Es kommt nun für die Trainingswissenschaft darauf an, ihre Legitimation auf der Basis eines interund inneruniversitären evaluativen Leistungsvergleichs nachzuweisen, was vielfach eine Adaptation an neue akademische Standards und Denkweisen (Internationalisierung und Vernetzung) erfordert.

Die beschriebenen Neuerungen an den Universitäten werden zum Anlass genommen, die Lage und Perspektiven der universitären Trainingswissenschaft in Form der vorliegenden Erklärung genauer zu analysieren. Sie benennt die spezifischen Problemlagen mit ihren Ursachen, die teilweise das Selbstverständnis der Disziplin berühren. Abschließend werden konkrete Maßnahmen gefordert, die ergriffen werden müssen, um die Zukunftsfähigkeit der Disziplin nachhaltig zu sichern.

\section{Zukunftsfähigkeit der Trainingswissenschaft}

Aus der vorgenommenen Zustandsbeschreibung können nachfolgende Merkmale des Markenkerns hervorgehoben werden, die im Hinblick auf die aktuellen akademischen Herausforderungen zukunftsfähige Entwicklungsperspektiven für die Trainingswissenschaft bieten.

\section{Eigene Forschungsstrategie}

Sowohl aus der grundsätzlichen Anwendungsorientierung als auch aus dem prozessualen Steuerungsgedanken von Training und Wettkampf selbst ergibt sich eine spezifische forschungsmethodische Herangehensweise an die Forschungsgegenstände Training, Leistungsfähigkeit und Wettkampf. Diese domainspezifische Forschungsstrategie manifestiert sich einerseits in einem feldorientierten, ganzheitlichen Zugang zu sportlichen Phänomenen und andererseits in einer an der Ziel- 
erreichung orientierten Schrittfolge von Ziel- und Prozessanalyse, Intervention, Kontrolle und Feedback.

Hierdurch hat sich in der Trainingswissenschaft eine mittlerweile hohe integrative Problemlösungskompetenz herausgebildet. Diese manifestiert sich auch im Sinne einer interdisziplinären Schnittstellenfunktion der Trainingswissenschaft in der Initiierung interdisziplinärer Theorieentwicklungs- und Problemlösungsstrategien. Die Trainingswissenschaft selbst verfügt hierbei neben eigenen disziplinären Kernkompetenzen insbesondere auch über fachliche Anschlusskenntnisse der kooperierenden Disziplin(en), die die Kommunikationsfähigkeit an den Disziplinschnittstellen sicherstellen.

Angesichts der zunehmenden Forderung nach interdisziplinärer Zusammenarbeit in der Bearbeitung komplexer Forschungsfragen, $z$. B. durch die forschungsfördernden Institutionen, erweist sich diese forschungsstrategische Entwicklung als äußerst zukunftsträchtig. Weiterhin eröffnet sie wichtige und konkurrenzfähige Entwicklungs- und Qualifizierungsperspektiven für den wissenschaftlichen Nachwuchs.

An dieser Stelle muss angemerkt werden, dass es bisher noch viel zu wenig gelungen ist, die Unterschiede zwischen dieser spezifisch trainingswissenschaftlichen Forschungsstrategie und denjenigen anderer Disziplinen zu verdeutlichen. Vielmehr begegnet man dort häufig einer Attitüde der Unterschätzung der damit verbundenen forschungsmethodischen und wissenschaftstheoretischen Anforderungen.

\section{Vielfältige Anwendungsfelder}

In den letzten Jahren hat die Trainingswissenschaft ihr Selbstverständnis in Richtung eines offenen Trainingsbegriffs entwickelt, wodurch neue Anwendungsfelder hinzugekommen sind. In Verbindung mit der zunehmenden Diversität der Studiengänge hat dies die Trainingswissenschaft als Lehrgebiet gestärkt. Mit der alleinigen Fokussierung auf das aus der Tradition erwachsene Anwendungsfeld „Leistungssport" wäre die umfassende Einbindung der Trainingswissenschaft in die Curricu- la womöglich weniger erfolgreich gelungen.

Gleichzeitig bieten die neuen Anwendungsfelder vielfältige Anknüpfungspunkte für trainingswissenschaftliche Fragestellungen, so dass auch auf dem Gebiet der Forschung in den letzten Jahren weitere Tätigkeitsbereiche erschlossen werden konnten. Die Öffnung des Trainingsbegriffes für neue Anwendungsfelder und Ziele darf allerdings nicht mit einer Beliebigkeit der wissenschaftlichen Arbeitsweise verwechselt werden, sondern muss sich stets an der spezifisch trainingswissenschaftlichen Forschungsstrategie orientieren.

\section{Spitzensportforschung}

Die Trainingswissenschaft trägt durch ihren Theorien- und Methoden-Vorlauf nicht unwesentlich zur Sicherung eines potenziellen Leistungsvorsprungs im Spitzensport bei. Es liegt auf der Hand, dass insbesondere die Universitäten als Orte der Spitzenforschung in dieser Hinsicht eine ganz zentrale Rolle spielen, wie sich leicht an den theoretischen Arbeiten beispielsweise zum nichtlinearen oder zum qualitativen Forschungsparadigma oder an den methodischen bzw. technologischen Entwicklungen in Bereichen wie der mathematischen Spielanalyse und Prozesssimulation, der automatischen Bild- und Mustererkennung oder auch der apparativen Muskelstimulation etc. festmachen ließe. Die Ergebnisse der trainingswissenschaftlichen Spitzensportforschung kommen dabei kurzfristig zunächst dem Spitzensport, mittel- und langfristig jedoch auch allen anderen Anwendungsfeldern des Sports zu Gute.

Die Anerkennung der Trainingswissenschaft im Bereich der Spitzensportforschung dürfte ganz wesentlich aus ihrer Innovationsfunktion in Verbindung mit ihrer ausgeprägten Integrationsfunktion resultieren. So übt die Trainingswissenschaft bedingt durch die dominante Anwendungsorientierung unter Berücksichtigung der ganzheitlichen Systemkomplexität von Training, Leistungsfähigkeit und Wettkampf eine Integrationsfunktion zwischen Wissenschafts- und Sportsystem aus. Auf Basis symmetrisch zirkulärer Dialoge mit Vertretern des Sports er- schließt sie drängende Problemlagen des Sports und löst diese kooperativ in praxisrelevanten Forschungszugängen, um anschließend die nachhaltige Implementierungen der Lösungen im Sport zu fördern und zu beurteilen. Wesentlich für diese systemübergreifende Schnittstellentätigkeit sind Anschlusskompetenzen aus dem Sport selbst, zu denen insbesondere detaillierte sportfachliche Kenntnisse, Kenntnisse über das Sportsystem und über die Sportorganisation zählen.

Die Verbindung von wissenschaftlicher Innovations- und systemübergreifender Integrationsfunktion stellt ein zukunftsfähiges Alleinstellungsmerkmal der Trainingswissenschaft dar, das seitens der Projektgeber in der Spitzensportforschung, wie auch allgemein in der Anwendungsforschung im Sport eine dauerhafte Nachfrage erfährt.

\section{Was sind aktuelle Probleme?}

Die Evaluation wissenschaftlicher Leistungen an den Universitäten ist ein typisches Merkmal der eingangs erläuterten Veränderungsprozesse an den Hochschulen. Verstärkt ist zu erwarten, dass aus diesen Leistungserfassungen konkrete strukturelle Entscheidungen abgeleitet werden, die in ihrer Summe eine gesamte Wissenschaftsdisziplin gefährden können, wenn es dieser nicht gelingt, den extern angelegten Maßstäben zu genügen.

Die bisherigen Erfahrungen deuten darauf hin, dass für eine nachhaltige Existenzsicherung vor allem die Leistungsindikatoren aus dem Bereich der Forschung, wie z. B. die Drittmitteleinwerbung, die besonders internationale - Publikationstätigkeit und die Anzahl der Promotionen und Habilitationen von besonderem Interesse sind. Aus Sicht der Trainingswissenschaft sollen nachfolgend potenzielle Problemlagen auf diesen Feldern identifiziert werden, die umso ernster zu nehmen sind, je mehr ihre Ursachen mit strukturellen oder sogar konstitutiven Merkmalen der Disziplin in Zusammenhang stehen.

\section{Drittmittel}

Die Einwerbung von Drittmitteln über disziplinübergreifende, hochkompetitive 
Fördereinrichtungen der Wissenschaft gestaltet sich unter Umständen problematisch, da die Trainingswissenschaft keine Verknüpfung mit einer einzigen, traditionell in den Förderstrukturen etablierten Bezugswissenschaft hat. Ein Blick beispielsweise auf die Struktur der DFG macht deutlich, dass die einzelnen Fächer und darüber hinaus auch die Fachkollegien entweder isolierte Teilgebiete der Basiswissenschaften (Medizin, Biologie, Physik usw.) repräsentieren oder zumindest stark mit einer der Basis- oder Bezugswissenschaft verknüpft sind. Ferner ist die im Vergleich zu anderen sportwissenschaftlichen Disziplinen starke Anwendungsperspektive in der trainingswissenschaftlichen Forschung weniger kompatibel mit den eher an der Grundlagenforschung orientierten Statuten dieser Forschungsfördereinrichtungen.

Die Leistungssportforschung ist das traditionelle Hauptbetätigungsfeld der Trainingswissenschaft. Zu den bereits genannten forschungsstrategischen Hindernissen (integrativ und anwendungsorientiert) kommt bei Forschungen in diesem speziellen Anwendungsfeld dazu, dass auf die Zuständigkeit des Bundesinstituts für Sportwissenschaft (BISp) verwiesen wird, dem die staatliche Forschungsförderung im Spitzensport einschließlich der Nachwuchsförderung obliegt.

Dem BISp kommt damit die Aufgabe $\mathrm{zu}$, die gesamte universitäre Leistungssportforschung aller sportwissenschaftlichen Disziplinen zu fördern. Zwar entfällt hier ein beträchtlicher Teil auf trainingswissenschaftliche Themen, jedoch ist die Projektdotierung in der klassischen Antragsforschung vergleichsweise bescheiden; größere Projektförderungen stellen bisher noch die Ausnahme dar.

Folgen sind sowohl die Orientierung auf neue Drittmittelgeber wie beispielsweise die Wirtschaft oder Einrichtungen des Gesundheitswesens unter Aufgabe der klassischen trainingswissenschaftlichen Fragestellungen als auch die Hinwendung zur Grundlagenforschung, womit häufig ebenfalls die trainingswissenschaftliche Perspektive das Nachsehen hat.

\section{Publikationen}

Was die bibliometrische Leistungsbewertung betrifft, so ist zunächst darauf hinzuweisen, dass die meisten der international renommierten und mit hohem ImpactFaktor versehenen Journals der Sportwissenschaft im angelsächsischen Sprachraum angesiedelt sind. Allerdings lassen sich in der angloamerikanischen Systematik der Sportwissenschaft erst neuerdings bedeutsame Entwicklungstrends hin zu einer eigenständigen Disziplin „Training Science“ oder „Coaching Science" und die Etablierung einer thematisch einschlägigen Publikationskultur feststellen. Auch oder gerade weil für die Trainingswissenschaft - im Gegensatz zu den meisten anderen sportwissenschaftlichen Disziplinen - eine internationale Wissenschaftsorganisation noch aussteht, darf die deutsche Trainingswissenschaft diesen internationalen Aufbruch nicht übersehen und muss ihre traditionell gewachsene hohe Expertise umfassend in die englischsprachige Diskussion einbringen.

In diesem Zusammenhang ist festzustellen, dass eine angewandte und ganzheitliche Ausrichtung von der Wissensproduktion bis hin zur Wissensanwendung, wie sie für die Trainingswissenschaft konstitutiv ist, von ausgewählten internationalen Journals zunehmend honoriert wird. Auch wenn sich wissenschaftliche Maßstäbe im Feld wesentlich schwieriger realisieren lassen als unter den kontrollierten Bedingungen des Labors, spricht dies für eine verstärkte Hinwendung zu feldnahen trainingswissenschaftlichen Themen und praxisorientierten Designs in der Forschung.

Für die Veröffentlichung trainingswissenschaftlicher Forschungsergebnisse auf international hohem Niveau haben sich die Rahmenbedingungen zuletzt durch die Etablierung neuer oder die Höherbewertung bestehender Journals (z. B. Journal of Sports Sciences, International Journal of Sport Science and Coaching, International Journal of Performance Analysis of Sport, High Ability Studies, The Journal of Strength and Conditioning Research, European Journal of Sport Science etc.) erheblich verbessert, auch wenn diese noch weiter ausbaufähig sind.

\section{Trainingswissenschaftlicher Nachwuchs}

Die bisher genannten Problembereiche Drittmittel und Publikationen bereiten besonders in der Ausbildung von trainingswissenschaftlichem Nachwuchs zusätzliche Probleme. Nachwuchswissenschaftler brauchen Projektstellen und Publikationsmöglichkeiten. Nach der Qualifikationsphase treten sie in Wettbewerb mit dem Nachwuchs anderer Disziplinen und müssen sich dort durchsetzen, da die Einstellungspraxis vermehrt von Publikationen und Projektakquisitionen bestimmt wird. Bedenkt man darüber hinaus, dass die für die Trainingswissenschaft typischen praktischen Interventionen meist nur mit einer langen Projektdauer auf der Basis einer persönlichen Vernetzung zu realisieren sind und dass wissenschaftliche Anforderungen in Einklang mit den Bedürfnissen der Sportpraxis gebracht werden müssen, dann ist der Aufwand für unseren Nachwuchs oft ungleich höher bei geringeren Erträgen.

Als Folge dieser Umstände ist es nicht verwunderlich, dass sich immer weniger Nachwuchs als klassischer Trainingswissenschaftler profilieren kann oder möchte. Damit droht schon in naher Zukunft, dass die Trainingswissenschaft ihre Stellen nicht mehr adäquat besetzen kann.

An dieser Stelle sei auf ein in der aktuellen Bildungslandschaft immer kritischer bewertetes Negativum hingewiesen, das sich in der Trainingswissenschaft recht drastisch darstellt. Sowohl bei den Hochschullehrern, wo nur eine der aktuell 33 Professuren mit einer Frau besetzt ist, als auch beim wissenschaftlichen Nachwuchs verfügt sie nur über einen marginalen Frauenanteil.

\section{Stellenbesetzungen}

Die Umorientierung der Universitäten bringt es mit sich, dass auch bei der Besetzung von Lehrstühlen und Professuren zunehmend die universellen Kriterien für Forschungsleistungen angelegt werden, so dass auch in dieser Hinsicht die oben genannten besonderen strukturellen Bedingungen der Trainingswissenschaft sich nachteilig auswirken. Dies führt da$\mathrm{zu}$, dass häufig die Trainingswissenschaft 
noch immer dominant als Lehraufgabe wahrgenommen wird, während sich Forschungsaktivitäten in anderen Disziplinen abspielen. Auch ist in vielen Besetzungsverfahren zu beobachten, dass zwar nominell das Fach „Trainingswissenschaft“ in Forschung und Lehre vertreten werden soll, dass aber die Sicherung der spezifischen trainingswissenschaftlichen Herangehensweise verglichen mit übergeordneten Kriterien oft ein vernachlässigtes Kriterium bei der Kandidatenauswahl spielt.

\section{Fazit}

Die geschilderten Problemlagen kann man in Teilen auch als "double bind“ interpretieren: Konstitutive Merkmale der Trainingswissenschaft wie die Praxisorientierung und das traditionelle Anwendungsfeld Leistungssport führen da$\mathrm{zu}$, dass die Kriterien, mit denen man Forschungsleistungen erfasst, schwieriger $\mathrm{zu}$ erfüllen sind. Stellt man sich jedoch diesen modernen Kriterien nicht oder unangemessen, so führt dies zu einer existenziellen Bedrohung der Disziplin. Unterwirft man sich vollständig diesen Kriterien, so bedroht oder verliert man gar seine Identität als Trainingswissenschaft!

\section{Maßnahmenkatalog zur Stärkung der Zukunftsfähigkeit der Trainingswissenschaft}

Die wichtigsten Maßnahmen zur Sicherung der Zukunftsfähigkeit der Disziplin sind zunächst die gezielte Ausrichtung auf die aus dem Markenkern abgeleiteten disziplinspezifischen Stärken (Forschungsstrategie, Anwendungsfelder und Spitzensportforschung) sowie deren fortwährender Ausbau. Darüber hinaus scheinen jedoch weitere konkrete Maßnahmen dringend erforderlich:

\section{Kommunikation des Markenkerns derTrainingswissenschaft}

- Kommunikation der Disziplinbesonderheiten gegenüber Vertretern von Forschungsfördereinrichtungen (BISp, BMI, DOSB, Sportfachverbände, DFG).

- Inneruniversitäre Kommunikation der Problemlagen der Disziplin, der Bedeutung der trainingswissenschaftlichen Lehre und der Bedrohung der gesamten Sportwissenschaft gegenüber den Universitätsleitungen, Strukturverantwortlichen, anderen Fächern und innerhalb der sportwissenschaftlichen Einrichtungen.

\section{Bessere Vernetzung}

- Verstärkung der Kooperationen mit Abnehmern und Partnern der universitären Trainingswissenschaft (Sportfachverbände, DOSB, OSP, IAT)

- Stärkung der Disziplin in internationaler Scientific Community (ECSS), z. B. durch Gründung einer eigenen Gruppe (Organisation/Sektion/Einheit)

\section{Verbesserungen in den Leistungskriterien}

- Vertretung der Trainingswissenschaft in internationalen Publikationsorganen

- Schaffung von modernen Publikationsmöglichkeiten für die deutsche Trainingswissenschaft (Englisch, Impact-Faktor)

\section{Personalförderung}

- Verstärkung/Intensivierung der gezielten Nachwuchsförderung (z. B. Selbstverpflichtung der Professoren, Veranstaltungen für den trainingswissenschaftlichen Nachwuchs)

- Gezielte Ausbildung des wissenschaftlichen Nachwuchses in Bezug auf die aus dem Markenkern abgeleiteten Stärken der Trainingswissenschaftlichen Forschung, z. B. bezüglich Entwicklung, Beantragung und Bearbeitung von interdisziplinären Forschungsprogrammen, Initiierung und Projektmanagement von Forschungsverbünden, disziplin- und systemübergreifende Schnittstellenkompetenzen

- Gezielte Maßnahme zur Erhöhung des Frauenanteils unter den Hochschullehrern und im wissenschaftlichen Nachwuchs

\section{Korrespondenzadresse}

Univ.-Prof. Dr. M. Lames

Lehrstuhl für Trainingswissenschaft

und Sportinformatik

Uptown München-Campus D

Georg-Brauchle-Ring 60/62, 80992 München

martin.lames@tum.de

Interessenkonflikt. Der korrespondierende Autor gibt für sich und seine Koautoren an, dass kein Interessenkonflikt besteht. 